\title{
Invited Discussion on: Non-surgical Rhinoplasty with Hyaluronic Acid Fillers: Predictable Results Using Software for the Evaluation of Nasal Angles
}

\author{
Ali Totonchi ${ }^{1}$
}

Received: 25 January 2020/Accepted: 28 January 2020/Published online: 18 February 2020

(C) Springer Science+Business Media, LLC, part of Springer Nature and International Society of Aesthetic Plastic Surgery 2020

Level of Evidence $V$ This journal requires that authors assign a level of evidence to each article. For a full description of these Evidence-Based Medicine ratings, please refer to the Table of Contents or the online Instructions to Authors www.springer.com/00266.

In this manuscript, the authors presented 62 patients who underwent nasal injections with hyaluronic acid-based injectables to improve the shape of the nose. The focus of the authors was to look for the safety of these injectables on the nose and whether these injectables can improve the frontonasal and nasolabial angles. Vycross injectables were used for this study.

Patients with deep nasion, nasal hump or under projected tip were selected for this study, and frontonasal and nasolabial angles were measured on the profile views and then analysed. The patients were told, and they agreed that their nose will become overall larger and the fact that they need to have realistic expectations. Patients with more than two rhinoplasties in the past, allergy to the studied fillers, excessive sun damage, wound healing problems, pregnancy or breast-feeding and history of nasal filler injections in the last 3 months were excluded.

All of the patients, except one, were followed up for 12 months, but nasal angles were measured for analysis only 1 month after the first injections. Twelve out of 62 patients required a second injection for touch-up, which I assume the second injection was done right before data collection on those 12 subjects. Pictures were taken before the initial injection and then 1 month after that they were assessed by "face master" software. In this study only,

Ali Totonchi

totonchimd@gmail.com

1 MetroHealth Medical Center, Case Western Reserve University, Cleveland, OH 44109, USA profile pictures were used and only two angles were measured (nasolabial and nasofrontal), and pre- and post-injection patient satisfaction rate as well as complications were also recorded.

The injection locations were limited to the dorsum of the nose and columella plus two microbolus to the tip using a 27-gauge needle from the base of the columella. Twelve out of 62 patients needed a touch-up injection with a smaller volume within the first month after the original injection. The average amount of injectable used in the first session was $0.55 \pm 0.22 \mathrm{ml}$ and $0.15 \pm 0.08 \mathrm{ml}$ 14-30 days after the original injection. Out of the total injected volume, almost $50 \%$ was injected on bony dorsum and the rest over the cartilaginous dorsum, tip and columella.

In my opinion, the strength of this manuscript is at presenting a method of injection to ensure patient safety and satisfaction, and this method starts with acknowledging the limitation of using injectables on the nose and selecting patients who are ok with having a larger nose and understand the limitations of this procedure. Dorsal hump and tip deficiency are the authors' indications for nasal correction with injectables as long as patients are fine to have a larger nose.

After reasonable patient selection, the authors stress the safety of injection by appropriate skin preparation with chlorhexidine. They also give us a few tips on how to respect the blood supply to the nose and adjusting the injection technique accordingly, limiting the volume of injection and if necessary staging it plus limiting the locations of the injection to a few areas and not using a cold compress are also mentioned in this manuscript as other safety measures. There are a few useful technical tips like blocking the nasal side wall during the injection to the bony nasal dorsum to block the shift of injectable to the 
nasal sidewall to prevent nasal widening and also gentle massage at the end to limit the chance of irregularity. These precautions are important in reducing the complications to a few transient ones like small hematoma, pain and swelling which was resolved in $48 \mathrm{~h}$ per authors.

On the other hand, the article has a few shortcomings which limit the clinical application of the manuscript to a narrow group of patients. Although the authors stated that the patients were followed for up to 11 months, there was no data collection beyond 1 month. They achieved the improved nasal angles and patient satisfaction within a month after injection with $20 \%$ of the patient needing a second injection, and we do not know if these results are sustained beyond the 30 days after the first injection.

The authors also did not exclude patients who had one rhinoplasty surgery. It would have been nice to exclude these patients to have a more homogenous study group. It is also not clear to us how many of these patients had surgery before injection, unanswered questions like, how this subgroup of patients responded to injections compared to patients who underwent injection with no previous surgery make the results less reliable. Since most of the patients who underwent rhinoplasty usually have improved dorsal hump and tip projection, the clear indication of filler injection in this subgroup of patients is not clear to the readers, considering that some clinicians use fillers for contour deformity in post-rhinoplasty patients which is a different indication than what is used in this study.

Standard photography was used with AP, lateral and oblique views, but only lateral views were used for analysis and we have no data collection on AP view, considering that nasal widening is a potential side effect of the fillers used on the nose.

Overall, the authors need to be congratulated on providing the readers with a few useful tips on the safety of the injectables on the nose and proper patient selection, but it would be useful if they also looked into other variables like the longevity of the results, how many of these patients ended up undergoing surgery, and the effects of the injectable on possible future rhinoplasty compared to primary rhinoplasty with no history of injection.

\section{Compliance with Ethical Standards}

Conflict of interest The author declares that he has no conflicts of interest to disclose.

Ethical Approval This article does not contain any studies with human participants or animals performed by any of the authors.

Informed Consent For this type of study, informed consent is not required.

Publisher's Note Springer Nature remains neutral with regard to jurisdictional claims in published maps and institutional affiliations. 